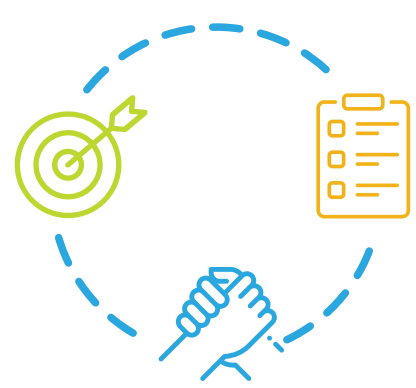

\title{
CONTRIBUIÇÕES DO APOIADOR DE PESQUISA E INTERVENÇÃO DO PROJETO SÍFILIS NÃO NA IMPLEMENTAÇÃO DE AÇÕES ESTRATÉGICAS NO ENFRENTAMENTO À SÍFILIS NO MUNICÍPIO DE ALVORADA/RS
}

CONTRIBUTIONS OF THE RESEARCH SUPPORTER AND INTERVENTION OF THE SYPHILIS PROJECT NOT IN THE IMPLEMENTATION OF STRATEGIC ACTIONS IN COPING WITH SYPHILIS IN THE MUNICIPALITY OF ALVORADA/RS

\section{Carla Zilio}

Administradora Hospitalar pela Universidade do Vale do Rio dos Sinos (UNISINOS). Especialista em Saúde Pública pela UFRGS e Gestão de Serviços Públicos de Saúde pelo IAHCS; Analista de Gestão em Saúde, no Instituto de Assistência à Saúde dos Servidores Públicos do Rio Grande do Sul. Apoiadora do Projeto de Pesquisa e Intervenção para Resposta Rápida à Sífilis nas Redes de Atenção à Saúde do

Ministério da Saúde e UFRN.

E-mail: carla.zilio@lais.huol.ufrn.br.

Sandra Regina Rocha Baldin

Enfermeira graduada pela UNISINOS. Especialista em Tuberculose pela FIOCRUZ; Enfermeira do Centro de Vigilância em Saúde da Secretaria Municipal de Saúde de Alvorada, responsável pelo Núcleo de Agravos e

Notificações Compulsórias.

E-mail: srbaldin@terra.com.br.

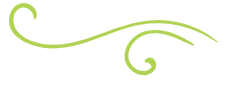

\section{RESUMO}

Descrever como foi implementado o Projeto Interfederativo de Resposta Rápida à Sífilis nas Redes de Atenção à Saúde, mais conhecido como Projeto "Sífilis Não", no município de Alvorada/RS. Consiste em um relato de experiência do apoiador de pesquisa e intervenção do Projeto "Sífilis Não" que busca elencar as principais atividades desenvolvidas pelo apoiador, no período de maio de 2018 a março de 2020, sustentada no referencial teórico do Apoio
Matricial e da Educação Permanente em Saúde. As atividades desenvolvidas contribuíram para a construção e o fortalecimento dos processos de trabalho relacionados à sífilis a partir da implementação de estratégias conduzidas pelo Comitê de Investigação da Transmissão Vertical de HIV, Sífilis e Hepatites Virais do município de Alvorada. A experiência relatada mostra que as estratégias promoveram ações conjuntas entre as áreas de vigilância e assistência, consolidando as 
ações do Comitê e a implementação da linha de cuidado da criança exposta à sífilis e com sífilis congênita.

Palavras-chave: Sífilis; Sífilis Congênita; Transmissão Vertical.

\section{ABSTRACT}

To describe how the Interfederative Project for Rapid Response to Syphilis was implemented in Health Care Networks, better known as the "No Syphilis Project", in the municipality of Alvorada/RS. This is an experience report of the research and intervention supporter of the Projeto Sífilis Não that seeks to list the main activities developed by the supporter from May 2018 to March 2020, supported by the theoretical framework of Matrix Support and Education Permanent in Health. The activities carried out contributed to the construction and strengthening of work processes related to Syphilis through the implementation of strategies conducted by the Investigation Committee for Vertical Transmission of HIV, Syphilis and Viral Hepatitis in the municipality of Alvorada. The reported experience shows that the strategies promoted joint actions between the areas of surveillance and assistance, consolidating the actions of the Committee and the implementation of the line of care for children exposed to syphilis and with congenital syphilis.

Key-words: Syphilis; Congenital Syphilis; Vertical Transmission.

\section{INTRODUÇÃO}

A Sífilis é uma Infecção Sexualmente Transmissivel (IST) provocada pela bactéria Treponema Pallidum. A principal via de transmissão é o contato sexual, seguido pela transmissão vertical para o feto durante o período de gestação de uma mãe com sífilis não tratada ou tratada inadequadamente (BRASIL, 2020a).
Segundo a Organização Mundial da Saúde (OMS), atinge mais de 12 milhões de pessoas em todo o mundo e sua eliminação continua a desafiar globalmente os sistemas de saúde (BRASIL, 2018b). No Brasil em 2019, foram notificados no Sistema de Informação de Agravos de Notificação (SINAN) 152.915 casos de sífilis adquirida (taxa de detecção de 72,8 casos/100.000 habitantes); 61.127 casos de sífilis em gestantes (taxa de detecção de 20,8/1.000 nascidos vivos); 24.130 casos de sífilis congênita (taxa de incidência de 8,2/1.000 nascidos vivos); e 173 óbitos por sífilis congênita (taxa de mortalidade de 5,9/100.000 nascidos vivos) (BRASIL, 2020b).

No Rio Grande do Sul (RS), em 2019, foram notificados 14.864 casos de sífilis adquirida, 4.594 casos de sífilis em gestantes, 1.839 casos de sífilis congênita e 8 óbitos por sífilis congênita (BRASIL, 2020b). Entre 2018 e 2019, observou-se que no RS ocorreu redução da taxa de detecção de sífilis adquirida de 134,8 para 130,6 casos por 100.000 habitantes. O mesmo processo não ocorreu com a taxa de detecção de sífilis em gestantes em que se observou um aumento de 28,6 para 32,8 casos por 100.000 habitantes. A taxa de incidência de sífilis congênita apresentou uma redução de 13,9 para 13,1 casos por 1.000 nascidos vivos.

No município de Alvorada, em 2019, foram notificados 312 casos de sífilis adquirida, 183 casos de sífilis em gestantes e 71 casos de sífilis congênita. Entre 2018 e 2019, ocorreu um aumento das taxas de detecção de sífilis em gestante e da taxa de incidência de sífilis congênita em Alvorada, já no número de óbitos por sífilis congênita, ocorreu uma redução de 04 óbitos em 2018 para nenhum óbito em 2019 (BRASIL, 2020c).

O efetivo controle da sífilis depende, em grande medida, da disposição e vontade política de gestores para colocar em prática um movimento em prol da qualidade da atenção à gestante e suas parcerias sexuais durante o pré-natal, promover mobilização nacional para ampliação do acesso ao diagnóstico da população geral e das populações-chave e 
estabelecer parcerias de base comunitária, além de vencer obstáculos quanto à administração de benzilpenicilina benzatina na Atenção Básica (AB) (BRASIL, 2018b).

Em resposta aos desafios para o controle da epidemia de sífilis, o Ministério da Saúde (MS) elaborou uma agenda de ações estratégicas para redução da sífilis no Brasil. Essa agenda resultou na iniciativa de uma emenda parlamentar de 200 milhões de reais, destinada a implementar um projeto de resposta rápida à sífilis em 100 municípios prioritários, que respondem por aproximadamente $65 \%$ dos casos de sífilis do país (BRASIL, 2018b). O projeto foi concebido para induzir ações voltadas ao controle da sífilis nas redes de atenção à saúde, com atuação de apoiadores locais; produzir conhecimentos por meio de estudos operacionais; e potencializar a capacidade técnica de vigilância e assistência locais (BRASIL, 2018b). Dessa forma, a inserção do apoiador do Projeto "Sífilis Não" no município de Alvorada/RS contribuiu para um diálogo efetivo entre as áreas técnicas, o desenvolvimento de estratégias alinhadas às necessidades da gestão e, principalmente, para dar visibilidade à sífilis como grave problema de saúde pública.

\section{DESENVOLVIMENTO}

O município de Alvorada está localizado na região metropolitana de Porto Alegre, no estado do RS, possui uma população estimada pelo Instituto Brasileiro de Geografia e Estatística (IBGE), em 2020, de 211.352 habitantes. Em 2017, o município apresentou taxas altas de detecção de sífilis em gestantes e adquirida e incidência de sífilis congênita, atendendo os critérios para seleção do Projeto Interfederativo de Resposta Rápida à Sífilis nas Redes de Atenção, considerando o número de habitantes e o índice composto de sífilis.

Em maio de 2018, ocorreu a primeira reunião com a participação da apoiadora de Pesquisa e Intervenção do Projeto "Sífilis
Não", no município de Alvorada, no RS. Naquela ocasião, foram apresentados os objetivos do Projeto e realizada a escuta dos coordenadores das áreas técnicas da Secretaria Municipal de Saúde (SMS) sobre as dificuldades e potencialidades relacionadas à sífilis. Nos primeiros meses, foi realizado o reconhecimento do território, conhecendo: a organização da rede municipal de saúde; os serviços ofertados; os profissionais das áreas técnicas; os fluxos assistenciais; a situação do Comitê de Investigação da Transmissão Vertical de HIV, Sífilis e Hepatites Virais; o cronograma de atividades de educação permanente; e os instrumentos de planejamento em saúde, com o objetivo de diagnosticar e elaborar um plano de trabalho para o município.

Ainda em 2018, as reuniões do Comitê de Investigação da Transmissão Vertical de HIV, Sífilis e Hepatites Virais contaram com a presença e as contribuições da apoiadora de pesquisa e intervenção, que, no mesmo ano, também realizou visitas, com a técnica do Centro Municipal de Vigilância em Saúde, às Unidades Básicas de Saúde para participar das reuniões de equipe para escuta dos profissionais de saúde que atuavam na assistência e para promover atividades para qualificação dos profissionais a partir das recomendações do Protocolo Clínico e Diretrizes Terapêuticas para Atenção Integral às Pessoas com Infecções Sexualmente Transmissíveis (PCDT-IST).

Nesse sentido, Campos (1999) menciona que o Apoio Matricial é uma metodologia de trabalho, um conjunto de conceitos sobre o "como fazer" o trabalho interprofissional, tanto em equipes quanto em redes de atenção à saúde (exercício da função apoio), em cogestão, de maneira compartilhada (CAMPOS, 1999). Nesse arranjo matricial, os profissionais das áreas especializadas que não participam integralmente do cotidiano das Equipes de Referências oferecem retaguarda especializada e suporte técnico-pedagógico a essas equipes (CUNHA; CAMPOS, 2011). 
Em 2019, foi proposto aos integrantes do Comitê a elaboração do relatório de recomendações para correção das falhas na assistência durante o pré-natal e seguimento da mulher após o parto, das crianças com sífilis congênita e exposta à sífilis, conforme o PCDT-IST. Durante as reuniões do Comitê, sempre foram discutidos os problemas observados nos estudos de casos que envolviam a qualidade da assistência ao pré-natal e a dificuldade dos profissionais de saúde em tomar a decisão adequada no manejo da sífilis em gestantes e na realização do monitoramento mensal das gestantes com sífilis. A partir disso, surgiu a ideia das recomendações aos serviços de saúde como uma solução alternativa para a superação dos problemas referentes à sífilis e, por conseguinte, a possibilidade de qualificar e aumentar a resolubilidade e a eficiência das redes de atenção à saúde.

Nesse contexto, a educação permanente em saúde é uma estratégia político-pedagógica que toma como objeto os problemas e necessidades emanados do processo de trabalho em saúde e incorpora o ensino, a atenção à saúde, a gestão do sistema e a participação e o controle social no cotidiano do trabalho com vista à produção de mudanças nesse contexto. Objetiva, assim, a qualificação e o aperfeiçoamento do processo de trabalho em vários níveis do sistema, orientando-se para a melhoria do acesso, a qualidade e a humanização na prestação de serviços e o fortalecimento dos processos de gestão político-institucional do Sistema Único de Saúde (SUS), no âmbito federal, estadual e municipal (BRASIL, 2018c).

Nessa perspectiva, as recomendações para correção dasfalhas na assistência durante o pré-natal e o seguimento da mulher após o parto, das crianças com sífilis congênita e expostas à sífilis se tornou um instrumento com a análise do caso e as indicações de conduta de tratamento e monitoramento. $\bigcirc$ documento passou a ser enviado pelo Centro Municipal de Vigilância em Saúde às equipes de Saúde da Família, Serviço Integrado da Mulher, Ambulatório de Gestação de Alto Risco e serviços privados visando orientar os profissionais de saúde em relação às condutas adotadas e com vista a evitar novos casos de transmissão vertical da sífilis.

Esse instrumento incentivou os profissionais de saúde preocupados com as falhas apontadas a buscarem orientações junto à referência técnica do Programa Municipal de IST/HIV/Aids e do Centro Municipal de Vigilância em Saúde. Paralelo a isso, as reuniões do Comitê continuaram acontecendo mensalmente e o grupo de trabalho foi ficando cada vez mais coeso e propositivo, sugerindo a participação de outras áreas técnicas, ampliando a discussão para a implementação das linhas de cuidado das crianças com sífilis congênita e expostas à sífilis, viabilizando mais uma estratégia para fortalecimento dos processos de trabalho no enfrentamento à sífilis.

A construção da Linha de cuidado da criança exposta à sífilis e com sífilis congênita foi resultado de inúmeros problemas observados nos estudos de casos analisados no Comitê e a constatação de que as crianças estavam recebendo alta hospitalar e as equipes de Saúde da Família não estavam realizando o seguimento clínico-laboratorial desses indivíduos. Outros problemas apontados foram: a baixa cobertura de atenção primária e, consequentemente, a falta de Unidade Básica de Saúde de referência para todos os casos; necessidade de realizar capacitação dos profissionais; falta de comunicação entre hospital e atenção primária; dificuldades de referenciamento para os serviços de saúde especializados; monitoramento laboratorial e de sinais e sintomas sugestivos de sífilis congênita até o $18^{\circ}$ mês, entre outros problemas percebidos pelo Comitê.

Frente a esses desafios, em que as práticas da Rede de Atenção à Saúde (RAS) estavam desarticuladas e prejudicando o seguimento das crianças com sífilis congênita e expostas à sífilis, foram implementadas as linhas de cuidado que expressam os fluxos assistenciais 
seguros e garantidos ao usuário, no sentido de atender as suas necessidades de saúde (FRANCO; FRANCO, 2012). Ademais, normatiza as ações que se desenvolvem em todos os pontos de atenção de uma RAS (níveis de atenção primária, secundária e terciária à saúde) e nos sistemas de apoio (MENDES, 2011).

\section{RESULTADOS E LIÇÕES APRENDIDAS}

A partir da compreensão da situação epidemiológica da sífilis do município de Alvorada e das dificuldades institucionais da SMS, a atuação do apoiador no território foi pautada no fortalecimento dos processos de trabalho, no estabelecimento de fluxos de atendimento, na integração das áreas técnicas e da rede de atenção à saúde, no papel do Comitê de Investigação da Transmissão Vertical de HIV, Sífilis e Hepatites Virais perante a essa grave situação de saúde pública que a sífilis se tornou, estimulando as práticas baseadas no PCDT-IST, busca ativa e monitoramento clínico-laboratorial dos casos. As atividades foram desenvolvidas em parceria com as referências técnicas municipais do Centro de Vigilância em Saúde, do Programa de IST/HIV/Aids, atenção básica, saúde da mulher e da coordenação médica e de enfermagem. Os encontros ocorreram periodicamente durante os quais eram discutidos os problemas e apontadas as possíveis soluções, considerando a estrutura organizacional do município.

No decorrer do desenvolvimento das atividades do Projeto, foi desafiador aprofundar vivências democráticas e de colaboração entre as áreas técnicas envolvidas. No início, a inserção junto às áreas técnicas da SMS e o trabalho do apoio avançaram lentamente, sendo essencial estabelecer relações de confiança com os profissionais das áreas técnicas. Após essa fase inicial, a articulação entre as áreas técnicas foi essencial para o funcionamento regular do Comitê e a construção dos demais instrumentos.
Ainda há muito o que progredir no enfrentamento à sífilis para reduzir o número de casos, visto que muitas intervenções não foram possíveis de realizar nesse período, pois demandam de mais tempo para planejamento e implementação.

\section{CONSIDERAÇÕES FINAIS}

A partir da narrativa apresentada, evidencia-se a necessidade de continuidade das ações até agora implementadas para alcançar os resultados almejados pelo Projeto "Sífilis Não". O vínculo estabelecido entre a apoiadora e as áreas técnicas favoreceu sobremaneira a organização das atividades e viabilizou um trabalho efetivo de apoio.

O apoiador de pesquisa e intervenção além de cumprir com um importante papel no acompanhamento e na organização dos processos de trabalho às áreas técnicas e assistenciais da SMS, precisa de muita capacidade de diálogo e escuta, disposição para articulação, além de qualificação técnica e muita disposição para novas experiências. É importante destacar que avanços são observados nesses dois anos, mas ainda há muito o que fazer, como ampliar os momentos de formação dos profissionais de saúde, a operacionalização das Linhas de Cuidado, articular ações intersetoriais entre saúde, controle social e comunidade, aumentar a cobertura de diagnóstico e fortalecer o processo de planejamento de ações em torno do enfrentamento da sífilis. Por fim, cabe reafirmar sobre a relevância na continuidade das ações estratégicas previstas no Projeto "Sífilis Não", possibilitando o fortalecimento e a qualificação dos processos de trabalho desenvolvidos pelo município, bem como dar seguimento ao trabalho coletivo e colaborativo do Comitê que possibilita o compartilhamento de conhecimentos e experiências. 


\section{REFERÊNCIAS}

BRASIL. Protocolo Clínico e Diretrizes Terapêuticas para Atenção Integral às Pessoas com Infecções Sexualmente Transmissíveis. Brasília: Ministério da Saúde, 2018a.

BRASIL. Protocolo Clínico e Diretrizes Terapêuticas para Atenção Integral às Pessoas com Infecções Sexualmente Transmissíveis (IST). Brasília: Ministério da Saúde, 2020a.

BRASIL. Ministério da Saúde. Boletim Epidemiológico Sífilis. Brasília, v. 49, n. 45, out. 2018b.

BRASIL. Ministério da Saúde. Boletim Epidemiológico Sífilis. Brasília, ano V., n. especial, out. 2019.

BRASIL. Ministério da Saúde. Boletim Epidemiológico Sífilis. Brasília, ano VI, n. especial, out. 2020b.

BRASIL. Ministério da Saúde. Política Nacional de Educação Permanente em Saúde: o que se tem produzido para o seu fortalecimento? 1. ed. rev. Brasília: Ministério da Saúde, 2018c.

BRASIL. Ministério da Saúde. Secretaria de Vigilância em Saúde. Departamento de Condições Crônicas e Infecções Sexualmente Transmissíveis. Indicadores e Dados Básicos da Sífilis nos Municípios Brasileiros, 2020c. Disponível em: http://indicadoressifilis.aids. gov.br/. Acesso em: 04 nov. 2020.

CAMPOS, G. W. S. Equipes de Referência e apoio especializado matricial: um ensaio sobre a reorganização do trabalho em saúde. Ciências Saúde Coletiva, v. 4, n. 2, p. 393-403, 1999.

CUNHA, G. T.; CAMPOS, G. W. S. Apoio Matricial e Atenção Primária em Saúde. Saúde e Sociedade, v. 20, n. 4, p. 961-970, dez. 2011.

FRANCO, C. M.; FRANCO, T. B. Linhas de cuidado integral: uma proposta de organização da rede de saúde. 2012. Disponível em: https://edisciplinas.usp.br/pluginfile.php/445762/ mod_resource/content/1/LINHAS_DO_CUIDADO_INTEGRAL.pdf. Acesso em: 11 mar. 2021.

MENDES, E. V. As redes de atenção à saúde. 2. ed. Brasília: Organização Pan-Americana da Saúde, 2011. 